\title{
Trend in initial presenting features of type 1 diabetes mellitus over a 24 year period in Turkey: a retrospective analysis of 814 cases
}

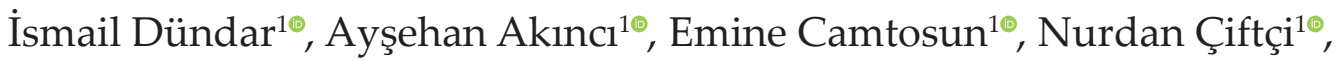 \\ Leman Kayas ${ }^{1 \oplus}$, Özlem Nalbantoğlu ${ }^{2}$ \\ ${ }^{1}$ Department of Pediatric Endocrinology, Inonu University Faculty of Medicine, Malatya; ${ }^{2}$ Department of Pediatric Endocrinology, \\ Izmir Provincial Health Directorate S.B.Ü. Dr.Behçet Uz Children's Education and Research Hospital, Izmir, Turkey.
}

\begin{abstract}
Background. The study aim was to examine changes in trends of presenting features during the diagnosis of patients followed up with newly diagnosed Type 1 diabetes mellitus (T1DM) over the past 24 years.

Methods. The study was retrospective. Patients with a diagnosis of T1D between the years of 1996-2019 were included. Patients diagnosed in the first half of the period comprised Period I, and those from the second half comprised Period II. Patient data were extracted from medical records and included gender distribution, year of diagnosis, age at diagnosis, duration of symptoms, type of admission, frequency of diabetic ketoacidosis (DKA) and biochemical parameters. Subsequently, temporal changes in trends of these parameters were sought.

Results. For the whole cohort the gender distribution was equal; 404 (49.6\%) were girls and 410 (50.4\%) were boys. Mean age at diagnosis was $8.5 \pm 4.2$ years and age groupings at presentation were: $23.2 \%(\mathrm{n}=189)$ aged $0-4 ; 39.2 \%(n=319)$ aged $5-9 ; 27.5 \%(n=224)$ aged $10-13 ; 10.1 \%(n=82)$ aged $14-18$. At presentation $72(12.7 \%)$ had hyperglycemia, $230(40.6 \%)$ had diabetic ketosis, and $264(46.6 \%)$ had DKA. In those with DKA, mild DKA was found in $103(39.0 \%)$, moderate DKA in $81(30.6 \%)$, and severe DKA in $80(30.3 \%)$. While the frequency of DKA was 54.9\% between 1996 and 2007 (Period I), this significantly decreased to 44.4\% between 2008 and 2019 (Period II). Girls and boys had a similar rate of T1DM, and this did not change over time. Three peak ages of diagnosis were evident; 5-7, 8-10, 12-14 years of age.

Conclusions. The frequency of DKA decreased and the frequency of admission with hyperglycemia and ketosis increased during the study period, which may have repercussions for mortality and morbidity rates and aid in improved treatment outcomes.
\end{abstract}

Key words: type I diabetes, diabetic ketoacidosis, childhood, trend.

Type 1 diabetes mellitus (T1DM) is an autoimmune disease that is usually diagnosed in childhood. T1DM is due to insulin deficiency caused by damage to pancreatic beta cells. ${ }^{1}$ Insulin deficiency will severely impair normal mechanisms of cellular glucose import. This results in a change in energy metabolism to lipid catabolism that may give rise to diabetic

$凶$ İsmail Dündar

ismail_dundar@yahoo.com

Received 11th December 2020, revised 8th July 2021, 13th August 2021, accepted 22nd October 2021. ketoacidosis. Several international registries have documented increasing rates of T1DM including DIAMOND, EURODIAB and SEARCH, with an annual increase in incidence of $2.8 \%, 3.5 \%$, and $2.72 \%$, respectively. ${ }^{2-4}$ The incidence of T1DM ranges from 0.7 to 40 per 100 thousand worldwide and has been shown to differ between communities and regions and within the same community in different ethnic groups. ${ }^{5}$ T1DM accounts for about $10 \%$ of all diabetes cases at any age. ${ }^{6}$ T1DM is more common in two periods, between the ages of 5-7 years, when school starts and the child may encounter novel infectious agents, and between 
the ages of 10-14 years when sex hormone concentrations increase during puberty which is accompanied by increased growth hormone concentrations and emotional stress is high. ${ }^{7,8}$

Diabetic ketoacidosis (DKA) is an acute complication of T1DM that usually occurs at the time of diagnosis, is life-threatening but rarely causes mortality. It has been reported that the frequency of DKA in patients with newly diagnosed T1DM varies widely from 15 to $80 \%$. ${ }^{9}$ ${ }^{11}$ Although there are Turkish national programs to decrease the frequency of DKA by increasing awareness of diabetes, it has been reported that there has been no significant decrease in the frequency of DKA at the time of diagnosis of T1DM cases in Turkey. ${ }^{12-14}$ The aim of this study was to evaluate the clinical and laboratory features of patients with newly diagnosed T1DM presenting to Inonu University Medical Faculty Hospital and Malatya Training and Research Hospital between the years of 19962019, and to investigate the trend of frequency of DKA over the years.

\section{Material and Methods}

Patient data were available from hospital records, which has been a source of reliable information about all diagnoses of pediatric T1DM since 1996. In Malatya, all children and young people (up to the age of 18 years) suspected of having newly diagnosed T1DM are seen at one of the two referral centers by specialists in pediatric endocrinology and diabetes. Each confirmed new case of T1DM is subsequently added to the registry. In addition, Syrian refugees residing in this region were also included in the study. Exclusion criteria included cases with syndromic diabetes, Type 2 diabetes (T2DM), maturity onset diabetes of youth (MODY), secondary causes of diabetes such as cystic fibrosis, steroid use and lipodystrophy, or those with missing data. As a result, a total of 814 T1DM cases diagnosed according to World Health Organization (WHO) criteria were included in the study.
Patients were categorized according to age: $<5$, 5-9, 10-13 and 14-18 years old. Patients with a diagnosis of T1DM between the years 19962019 were included. Patients diagnosed in the first half of the period comprised Period I, and those from the second half comprised Period II. Hyperglycemia was defined as plasma glucose $(\mathrm{PG}) \geq 200 \mathrm{mg} / \mathrm{dL}$. Confirmation of the diagnosis of T1DM was dependent on the presence of hyperglycemia, reliance on continued insulin treatment and presence of typical symptoms of T1DM at presentation. DKA was defined as hyperglycemia concurrent with acidosis (venous blood $\mathrm{pH} 7.30$ and/or serum bicarbonate $15 \mathrm{mmol} / \mathrm{L}$ ), ketonemia, and/or ketonuria. In addition presenting characteristics were divided into three clinical categories: 1 ) hyperglycemia without ketosis or acidosis; 2), hyperglycemia with ketosis but without acidosis; and 3) ketoacidosis. For the purposes of this study, ketosis assessment was performed by measuring urinary ketone semi-quantitatively. Thus ketonuria was present when urine ketone was $\geq 2+$. Urinary ketone measurements were performed by an automated analyzer which used sodium nitroprusside reaction as a test principle (BT URICELL 1280-1600 devices (BT products, İzmir, Turkey) in each voiding. DKA severity was classified according to the Lawson Wilkins Pediatric Endocrine Society Consensus Statement ${ }^{15}$, as follows: severe DKA (venous $\mathrm{pH}$ : $<7.10$; serum bicarbonate $<5 \mathrm{mmol} / \mathrm{L}$ ), moderate DKA (venous $\mathrm{pH}$ : 7.10 - 7.19; serum bicarbonate between 5 and $10 \mathrm{mmol} / \mathrm{L}$ ), and mild DKA (venous $\mathrm{pH}$ : 7.20 - 7.29; serum bicarbonate between 10 and 15 $\mathrm{mmol} / \mathrm{L}$ ). Demographic and clinical features at the time of diagnosis, including gender, birth date, diagnosis date, the season at presentation, duration of symptoms prior to diagnosis, and type of presentation (DKA, hyperglycemia with ketosis or hyperglycemia only) were collected. In addition, laboratory results including venous blood glucose concentration, c-peptide concentration, hemoglobin A1c $(\mathrm{HbA1c})$ percentage and the presence of T1DMassociated autoantibodies such as anti-glutamic 
acid decarboxyase antibody (GAD), insulin antibody (IAA) and islet cell antibody (ICA) were recorded from patient files. ICA, IAA, and GADA levels were measured using an enzymelinked immunosorbent assays based on antigenantibody detection with the Isletest commercial kit in the Seac Brio 410499 model instrument. The HbA1c level was measured by high performance liquid chromatography using an Agilent 1100 model instrument at our hospital. Ethics Committee Approval was obtained from the Malatya Training and Research Hospital Ethics Committee for the study (approval date: 18.11.2019; approval number: 23536505-604.02).

\section{Statistical analysis}

The data are presented as mean \pm standard deviation (SD) values. Data analysis was performed using SPSS for Windows statistical software, version 17.0 (SPSS, Chicago, IL, USA). Student's two-tailed t-test was used for comparisons between independent variables with a normal distribution. Mann-Whitney U-test was used for variables showing uneven distribution. Distribution was analyzed by cross-tabulation and Chi-square statistics. A p-value of $<0.05$ is considered to be statistically significant.

\section{Results}

The cohort consisted of 814 patients between the ages of $0.4-18$ years. Four hundred and four were female (49.6\%) and 410 were male. Across the whole cohort the mean age at diagnosis was $8.5 \pm 4.2$ years with a range of $0.37-17.52$ years. When patients were divided into four groups by age of diagnosis there were 189 patients (23.2\%) between 0-4 years old, 319 patients (39.2\%) between 5-9 years old, 224 patients $(27.5 \%)$ between $10-13$ years old and 82 patients $(10.1 \%)$ between 14-18 years old. There were three peak ages at diagnosis, between the ages of 5-7, 8-10 and 12-14 years. When seasonality of diagnosis was examined $28.4 \%$ of the patients were diagnosed in winter, $26.7 \%$ in autumn, $24.2 \%$ in spring and $20.8 \%$ in summer.
The mean duration of symptoms prior to diagnosis (polyuria, polydipsia, nocturia) was $18.7 \pm 18$ days (range 2-90). At the time of diagnosis, mean blood glucose concentration was $478 \pm 187 \mathrm{mg} / \mathrm{dL}$ (range: 128-1173), mean C-peptide was $0.6 \pm 0.5(0.1-3.8) \quad \mathrm{ng} / \mathrm{mL}$ and mean $\mathrm{HbA} 1 \mathrm{c}$ was $12.4 \pm 2.7 \%$ (range: $6.0-22.4 \%$ ). At diagnosis autoantibodies were assessed in $513(63 \%)$ cases. The antibody test records of the remaining 301 patients were not available. Serum autoantibody levels of 87 patients in Period I and 426 patients in Period II were evaluated. GAD (62.40\%) being most common followed by ICA (45.2\%) and IAA (29.7\%). Although at least one of the three antibodies was positive in 438 (85.4\%) of these cases, none of the three antibodies was detected in 75 $(14.6 \%)$ of the cases. Frequency of autoantibody positivity changed between Period I and Period II; ICA positivity increased from 20.7 to $50.0 \%$ $(\mathrm{p}<0.001)$ and IAA decreased from 44.8 to $26.5 \%$ $(\mathrm{p}<0.001)$ in Period I and Period II, respectively (Table I).

In 566 cases, clinical presentation at diagnosis was evaluated (122 patients in Period I and 444 patients in Period II). The records of the remaining 248 patients were not available. Of the 566 patients for whom data was available, 72 cases $(12.7 \%)$ presented with hyperglycemia, 230 cases $(40.6 \%)$ hyperglycemia with ketosis, and 264 cases $(46.6 \%)$ with DKA. Of those with DKA 103 (39.0\%) had mild DKA, 81 (30.6\%) had moderate DKA and 80 (30.3\%) had severe DKA. Key features of presentation were compared between Period I and Period II. In Period I 54.9\% of patients presented with DKA. In Period II this dropped and plateaued at $44.4 \%$ after 2007. Over the years, the rate of presentation with DKA has declined (Fig. 1). The frequency of DKA subgroups did not change between periods. Further comparisons between Period I and Period II were made (Table I). Clinical characteristics of our cases according to age groups are shown in Table II. 
Table I. Comparison of clinical and laboratory findings in type 1 diabetes by year of presentation.

\begin{tabular}{|c|c|c|c|}
\hline & Period 1 (1996-2007) & Period 2 (2008-2019) & $p$ value \\
\hline Number of the patients & 234 & 580 & \\
\hline Age (years) & $7.6 \pm 3.7$ & $8.9 \pm 4.28$ & $<0.05$ \\
\hline \multicolumn{4}{|l|}{ Gender n, (\%) } \\
\hline Female & $122(52.1)$ & $282(48.6)$ & NS \\
\hline Male & $112(47.9)$ & $298(51.4)$ & NS \\
\hline \multicolumn{4}{|l|}{ Age Groups n, (\%) } \\
\hline Ages of $0-4$ years & $67(28.6)$ & $122(21.0)$ & 0.020 \\
\hline Ages of 5-9 years & $95(40.6)$ & $224(38.6)$ & NS \\
\hline Ages of $10-13$ years & $63(26.9)$ & $161(27.8)$ & NS \\
\hline Ages of $14-18$ years & $9(3.85)$ & $73(12.7)$ & $<0.001$ \\
\hline Duration of symptoms before diagnosis (days) & $18.9 \pm 16.4$ & $18.6 \pm 18.7$ & NS \\
\hline Glucose (mg/dL) & $465.6 \pm 193$ & $483 \pm 185$ & NS \\
\hline C-peptide (ng/mL) & $0.6 \pm 0.4$ & $0.6 \pm 0.5$ & NS \\
\hline $\operatorname{HgA1C}(\%)$ & $13.3 \pm 3.5$ & $12.0 \pm 2.4$ & $<0.001$ \\
\hline \multicolumn{4}{|l|}{ Main presenting feature } \\
\hline Hyperglycemia n, (\%) & $11(9.0)$ & $61(13.7)$ & NS \\
\hline Ketosis $n,(\%)$ & $44(36.1)$ & $186(41.9)$ & NS \\
\hline Ketoacidosis n, (\%) & $67(54.9)$ & $197(44.4)$ & 0.039 \\
\hline \multicolumn{4}{|l|}{ DKA } \\
\hline Mild DKA n, (\%) & $22(32.8)$ & $81(41.1)$ & NS \\
\hline Moderate DKA n, $(\%)$ & $20(29.9)$ & $61(31.0)$ & NS \\
\hline Severe DKA n, $(\%)$ & $25(37.3)$ & $55(27.9)$ & NS \\
\hline \multicolumn{4}{|l|}{ Autoantibody positivity } \\
\hline Anti-GAD positivity $n,(\%)$ & $56(64.4)$ & $262(61.5)$ & NS \\
\hline ICA positivity $n,(\%)$ & $18(20.7)$ & $213(50.0)$ & $<0.001$ \\
\hline IAA positivity $n,(\%)$ & $39(44.8)$ & $113(26.5)$ & $<0.001$ \\
\hline
\end{tabular}

GAD: Glutamic acid decarboxylase antibody, IAA: Insulin antibody, ICA: Islet cell antibody, HgA1C: Glycosylated hemoglobin, DKA: Diabetic ketoacidosis

NS: Not significant.

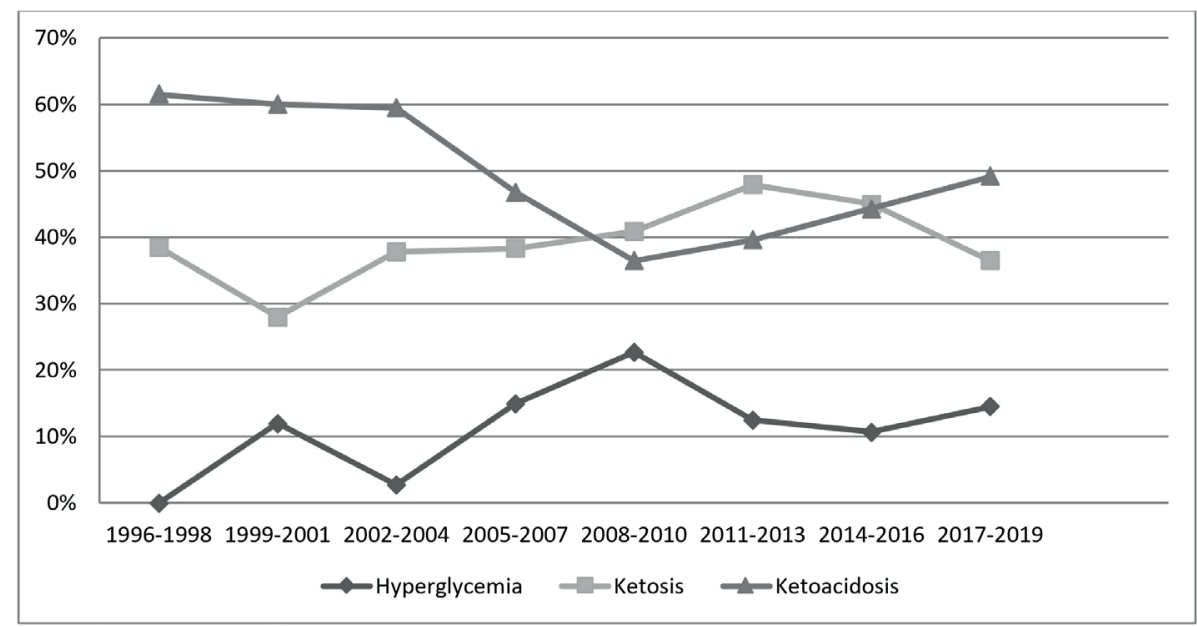

Fig. 1. Change of admission characteristics of patients with type 1 diabetes mellitus over the years. 
Table II. Clinical characteristics of cases according to age groups.

\begin{tabular}{llllll}
\hline Age group (years) & $0-4$ & $5-9$ & $10-13$ & $14-18$ & Total (0-18) \\
\hline Main presenting feature (n=566) & & & & & \\
$\quad$ Hyperglycemia n, (\%) & $9(7.0)$ & $24(11.7)$ & $30(18.4)$ & $9(12.9)$ & $72(12.7)$ \\
$\quad$ Ketosis n, $(\%)$ & $59(46.1)$ & $78(38.0)$ & $58(35.6)$ & $35(50.0)$ & $230(40.6)$ \\
$\quad$ Ketoacidosis n, (\%) & $60(46.9)$ & $103(50.2)$ & $75(46.0)$ & $26(37.1)$ & $264(46.6)$ \\
Severity of DKA (n=264) & & & & & \\
$\quad$ Mild DKA n, (\%) & $27(45.0)$ & $44(42.7)$ & $23(30.6)$ & $9(34.6)$ & $103(39.0)$ \\
$\quad$ Moderate DKA n, (\%) & $10(16.6)$ & $31(30.0)$ & $31(41.4)$ & $9(34.6)$ & $81(30.6)$ \\
$\quad$ Severe DKA n, $(\%)$ & $23(38.3)$ & $28(27.3)$ & $21(28.0)$ & $8(30.7)$ & $80(30.3)$ \\
\hline
\end{tabular}

DKA: Diabetic ketoacidosis

\section{Discussion}

Incidence of T1D is increasing all over the world. ${ }^{2-4}$ T1DM is one of the most common chronic endocrine diseases of childhood and adolescence and its incidence has been reported to vary with age, ethnicity, geographical region and season. ${ }^{16}$ When the gender distribution of our diabetic patients was examined, the female/ male ratio was 0.98 with no significant gender bias $(p=0.64)$. Although autoimmune diseases tend to be more common in girls compared to boys, there is no gender difference in children and adolescent T1DM cases ${ }^{17}$, in keeping with our findings. The mean age of diagnosis in our cohort was found to be $8.5 \pm 4.1$ years. Poyrazoğlu et al. ${ }^{18}$ in a study of T1DM patients aged $<18$ years reported a mean age of diagnosis of 9.2 years. Ardicli et al. ${ }^{14}$ conducted a study between the years 1969-1991 and the average age of diagnosis was found to be 9.5 years. In our study, the average age of diagnosis between 2008 and 2019 had increased significantly to 8.9 years. The reason for this may be that the oldest age group (14-18 years) only made up 3.8\% of all diagnoses in the earlier period of our study but this increased three-fold $(12.7 \%)$ in the period between 2008-2019. Similarly, in the present study, diagnosis of cases in the youngest age group (0-4 years) fell from $28.6 \%$ in the earlier period to $21 \%$ between 2008 and 2019.

T1DM is generally reported to exhibit a bimodal distribution in age of diagnosis, with peaks at the age of 5-7 years and puberty. The first peak is thought to occur due to common infections, and the second peak occurring during puberty is due to stress and increased concentrations of circulating gonadal hormones. ${ }^{19,20}$ In a multicenter study conducted by the European Diabetes Study Group ${ }^{21}$ on 15,000 diabetics, when patients were divided into three groups (0-4 years, 5-9 years, and 10-14 years) the frequency of diabetes was $24 \%, 35 \%$, and $41 \%$, respectively, increasing with age. Demir et al. ${ }^{12}$ reported that T1DM was seen in two separate age groups, most frequently between the ages of 6-8 and the second most frequently between 11-12. In our study group, the frequencies of diabetes in $0-4,5-9,10-13,14-18$ age groups were $23.2 \%, 39.2 \%, 27.5 \%$ and $10.1 \%$, respectively. In addition, the frequency of the occurrence of T1DM in our study was found to peak in three different age groups, 5-7, 8-10 and 12-14 years old. While the first peak was detected in early childhood, the second peak was detected in early puberty and the third peak in late puberty.

First presentation with T1DM has been reported to be seasonal. Seasonal variations in onset of T1DM were reported in many populationbased studies. ${ }^{22,23}$ Similarly, in our study, it was observed that most presentations occurred in winter and the lowest number was in summer. The increasing frequency of viral infections in winter is thought to trigger the emergence of diabetes by increasing the need for insulin. The average symptom duration prior to diagnosis has been reported as 14 days from studies in Australia and Sweden ${ }^{24,25}$ in patients with new diagnosis T1DM. In our study this was found to 
be slightly longer at 17.8 days. In our opinion this stems from the relatively poorer socioeconomic level of our region, more difficult accessibility to health institutions, cultural differences and geographical factors.

In T1DM, the destruction of pancreatic islet beta cells occurs through $\mathrm{T}$ cell-mediated cellular mechanisms in $80-90 \%$ of patients. However, autoantibodies are present at diagnosis in a significant proportion of patients. Kong et al. ${ }^{26}$ detected at least one of three antibodies in $71.4 \%$ of patients during the diagnosis of type 1 diabetic patients, including GAD in $66.2 \%$, ICA in $54.1 \%$ and IAA in $35.6 \%$ in patients who were antibody positive. Demir et al. $^{12}$ found GAD $(70 \%)$ antibodies to be most common, then ICA $(44.4 \%)$ and IAA (42.6\%), respectively, in patients with T1DM. In our study at least one of these three antibodies were positive in $85.4 \%$ of the cases, with GAD (62.40\%) being most common followed by ICA $(45.2 \%)$ and IAA $(29.7 \%)$. When the rates of antibody positivity were compared between Period I and Period II there was no difference in GAD positivity while ICA positivity significantly increased from $20.7 \%$ to $50.0 \%$ and, at the same time, the IAA decreased significantly from $44.8 \%$ to $26.5 \%$. The reason for this change has not been fully explained.

The first clinical presentation of patients with T1DM might range from hyperglycemia to diabetic coma. Demirbilek et al. ${ }^{13}$ reported the frequency of DKA at first presentation to be $65.0 \%$ in their study between the years 20102011. Ardicli et al. ${ }^{14}$ reported the frequency of diagnosis with DKA to be $50.5 \%$ between 1990-2000 and 51.0\% between 2000-2010. Acar et al. $^{27}$ found the incidence of DKA to be $43.2 \%$ in patients with a new diagnosis of T1DM between 1999 and 2014. In our cohort the overall rate of DKA presentation was $46.6 \%$, which is consistent with earlier Turkish reports. The frequency of DKA is lower in countries where T1DM is more frequent, such as Finland $(22.0 \%)$, Sweden $(14.0 \%)$, and Canada $(18.6 \%)$, whereas T1DM prevalence is higher in countries with low prevalence including the
Arab peninsula (80.0\%), Romania $(67.0 \%)$, and Taiwan $(65.0 \%) .{ }^{11}$ It was reported that rates of DKA ranged between 26 and $67 \%$ in a 24 -center study of the European Diabetes Study Group ${ }^{28}$, covering 1260 cases. In the same study, it was highlighted that the incidence of DKA was higher in patients aged under 5 years of age compared to previous years. In New Zealand, Jackson et al..$^{29}$ reported that the frequency of DKA, which they monitored over an eight-year period, decreased from 63 to $42 \%$, and that the blood glucose and $\mathrm{HbA1c}$ values in the newly diagnosed patient group were significantly lower compared to previous years. As a result of our 24-year follow-up it was found that the frequency of DKA decreased, and the frequency of admissions with hyperglycemia and ketosis increased. In Period I the frequency of presentation with DKA was $54.9 \%$, which decreased to $44.4 \%$ in Period II. There is a need to increase both public awareness and to ensure that physicians are alert to the possibility of T1DM, even in young patients.

The severity of DKA at presentation has been previously investigated. Acar et al. ${ }^{27}$ found severe DKA in $22.1 \%$, moderate in $32.8 \%$ and mild in $45.1 \%$ at the time of first presentation. In a study from Germany ${ }^{30}$, the majority of these cases $(63.8 \%)$ had no ketoacidosis, while $16.6 \%$ had mild, $12.1 \%$ moderate, and $4 \%$ severe ketoacidosis at the time of diagnosis. These rates in our cohort were $30.3 \%, 30.6 \%$ and $39.0 \%$ for severe, moderate and mild DKA, respectively. In addition, the frequency of severe DKA was higher in the $0-4$ years-old age group. The frequency of presentation with severe DKA was higher in the earlier period compared to the later period, $34.8 \%$ vs $26.3 \%$.

The decrease in frequency of first presentation with both DKA and severe DKA was interpreted as a positive development towards likely decreased mortality and morbidity. However, in Turkey, evidence from multiple studies have shown that the frequency of DKA at first presentation is still high and has variable outcome. We recommend multicentre studies to clearly determine the national frequency 
of DKA. This will provide the foundation for development of measures, involving pediatric endocrinologists, pediatricians and family physicians, in order to increase general public awareness of the first signs and symptoms of diabetes and improve the duration between first signs and presentation to a suitable specialist for assessment. For this purpose, joint seminars, public meetings, and training days in schools can be organized. In addition, social media, which is both ubiquitous and an effective communication tool, should be used. Furthermore, improved accessibility to health centers and increased socioeconomic opportunities will encourage more rapid patient presentation.

This study has some limitations. In this retrospective study, a number of factors which may have affected our results were not assessed. These included family socioeconomic status and parental education level, the status of refugees, the ease of availability of health care, the number of presentations before definite diagnosis, the type of hospital used for first presentation, the status of the pediatric endocrinology centers in the region, and the number of pediatricians involved.

The rate of first presentation with DKA in T1DM cases decreased at our city between the years 1996-2019. The proportion of patients presenting with severe DKA did not change. Interestingly and in contrast to most reports, the proportion of patients presenting in the earliest age group, 0-4 years also decreased while the proportion of patients presenting in late adolescence increased. This resulted in the average age of diagnosis increasing and, as younger patients are more likely to present with DKA, may have influenced the decreasing rate of DKA at presentation. There were three peak ages of diagnosis evident in our cohort; at the ages of $5-7,8-10$, and 12-14 years. It is suggested that the rate of DKA at presentation remains a problem in Turkey, as has previously been shown by several studies.

\section{Ethical approval}

Ethics Committee Approval was obtained from the Malatya Training and Research Hospital Ethics Committee for the study (approval date: 18.11.2019; approval number: 23536505-604.02).

\section{Author contribution}

The authors confirm contribution to the paper as follows: study conception and design: ID, NÇ, AA, EÇ; data collection: ID, NÇ; analysis and interpretation of results: ID, NÇ, AA, EÇ, LK, ÖN; draft manuscript preparation: ID, NÇ, AA, EÇ, LK, ÖN. All authors reviewed the results and approved the final version of the manuscript.

\section{Source of funding}

The authors declare the study received no funding.

\section{Conflict of interest}

The authors declare that there is no conflict of interest.

\section{REFERENCES}

1. Chiang JL, Kirkman MS, Laffel LM, Peters AL. Type 1 diabetes through the life span: a position statement of the American Diabetes Association. Diabetes care 2014; 37: 2034-2054. https://doi.org/10.2337/dc141140

2. The DIAMOND Project Group. Incidence and trends of childhood type 1 diabetes worldwide 1990-1999. Diabet Med 2006; 23: 857-866. https://doi. org/10.1111/j.1464-5491.2006.01925.x

3. Patterson C, Rosenbauer J, Neu A, et al. Incidence trends for childhood type 1 diabetes during1989-2013 in 24 European registries participating in the EURODIAB study. Pediatr Diabetes 2016; 17: 10-35. https://doi.org/10.1111/pedi.12450

4. Lawrence JM, Imperatore G, Dabelea D, et al. Trends in incidence of type 1 diabetes among non-Hispanic white youth in the US, 2002-2009. Diabetes 2014; 63: 3938-3945. https://doi.org/10.2337/db13-1891 
5. Sperling MA, Tamborlane WV, Battelino T, Weinzimer SA, Phillip M. Diabetes mellitus. In: Sperling, MA (ed). Pediatric Endocrinology (4th ed). Philadelphia, PA: Elsevier/Saunders, 2014: 846-900. https://doi.org/10.1016/B978-1-4557-4858-7.00028-7

6. Svoren BM, Jospe N. Diabetes mellitus. In: Kliegman RM, Stanton BF, St Geme III JW, Schor NF, Behrman RE, (eds). Nelson Textbook of Pediatrics (20th ed) Vol 2. Philadephia: Elsevier, 2016: 2760-2790.

7. Becker DJ. Complications of insulin dependent diabetes mellitus in childhood and adolescence. In: Lifshitz F (ed). Pediatric Endocrinology (3th ed). New York and Basel: Marcel Decker Inc, 1996: 583598.

8. Arslanian S, Drash AL. Insulin-dependent diabetes mellitus in children and adolescents. Curr Ther Endocrinol Metab 1994; 5: 380-384.

9. Rewers A, Klingensmith G, Davis C, et al. Presence of diabetic ketoacidosis at diagnosis of diabetes mellitus in youth: the Search for Diabetes in Youth Study. Pediatrics 2008; 121: e1258-e1266. https://doi. org/10.1542/peds.2007-1105

10. Levy-Marchal C, Patterson CC, Green A; EURODIAB ACE Study Group. Europe and Diabetes. Geographical variation of presentation at diagnosisof type I diabetes in children: the EURODIAB study. European and Dibetes. Diabetologia 2001; 44: 75-80. https://doi.org/10.1007/PL00002958

11. Usher-Smith JA, Thompson M, Ercole A, Walter FM. Variation between countries in the frequency of diabetic ketoacidosis at first presentation of type 1 diabetes in children: a systematic review. Diabetologia 2012; 55: 2878-2889. https://doi. org/10.1007/s00125-012-2690-2

12. Demir F, Günöz H, Saka N, et al. Epidemiologic features of type 1 diabetic patients between 0 and 18 years of age in İstanbul city. J Clin Res Pediatr Endocrinol 2015; 7: 49-56. https://doi.org/10.4274/ jcrpe. 1694

13. Demirbilek H, Ozbek MN, Baran RT. Incidence of type 1 diabetes mellitus in Turkish children from the southeastern region of the country: a regional report. J Clin Res Pediatr Endocrinol 2013; 5: 98-103. https://doi.org/10.4274/Jcrpe.954

14. Ardicli D, Kandemir N, Alikasifoglu A, Ozon A, Gonc N. Clinical characteristics of type 1 diabetes over a 40 year period in Turkey: secular trend towards earlier age of onset. J Pediatr Endocrinol Metab 2014; 27: 635-641. https://doi.org/10.1515/ jpem-2013-0320
15. Dunger DB, Sperling MA, Acerini CL, et al. European Society for Paediatric Endocrinology/Lawson Wilkins Pediatric Endocrine Society consensus statement on diabetic ketoacidosis in children and adolescents. Pediatrics 2004; 113: e133-e140. https:// doi.org/10.1542/peds.113.2.e133

16. Forouhi NG, Wareham NJ. Epidemiology of diabetes. Medicine (Abingdon) 2014; 42: 698-702. https://doi.org/10.1016/j.mpmed.2014.09.007

17. Maahs DM, West NA, Lawrence JM, Mayer-Davis EJ. Epidemiology of type 1 diabetes. Endocrinol Metab Clin North Am 2010; 39: 481-497. https://doi. org/10.1016/j.ecl.2010.05.011

18. Poyrazoğlu Ş, Bundak R, Yavaş Abalı Z, et al. Incidence of type 1 diabetes in children aged below 18 years during 2013-2015 in northwest Turkey. J Clin Res Pediatr Endocrinol 2018; 10: 336-342. https://doi.org/10.4274/jcrpe.0025

19. Pundziūtè-Lyckå A, Urbonaitė B, Dahlquist G. Infections and risk of Type I (insulin-dependent) diabetes mellitus in Lithuanian children. Diabetologia 2000; 43: 1229-1234. https://doi. org/10.1007/s001250051517

20. Shashaj B, Sulli N. Difference in insulin usage patterns with pubertal development in children with type 1 diabetes during transition from multiple daily injections to continuous subcutaneous insülin infusion (CSII) and through the CSII treatment. Diabetes Technol Ther 2009; 11: 767-774. https://doi. org/10.1089/dia.2009.0049

21. Patterson CC, Dahlquist GG, Gyürüs E, Green A, Soltész G; EURODIAB Study Group. Incidence trends for childhood type 1 diabetes in Europe during 1989-2003 and predicted new cases 2005-20: a multicentre prospective registration study. Lancet 2009; 37: 2027-2033. https://doi.org/10.1016/S01406736(09)60568-7

22. Moltchanova EV, Schreier N, Lammi N, Karvonen $\mathrm{M}$. Seasonal variation of diagnosis of type 1 diabetes mellitus in children worldwide. Diabet Med 2009; 26: 673-678. https://doi.org/10.1111/j.14645491.2009.02743.x

23. Dahlquist G, Mustonen L. Childhood onset diabetes--time trends and climatological factors. Int J Epidemiol 1994; 23: 1234-1241. https://doi. org/10.1093/ije/23.6.1234

24. Bui TP, Werther GA, Cameron FJ. Trends in diabetic ketoacidosis in childhood and adolescence: a 15-yr experience. Pediatr Diabetes 2002; 3: 82-88. https:// doi.org/10.1034/j.1399-5448.2002.30204.x 
25. Hanas R, Lindgren F, Lindblad B. Diabetic ketoacidosis and cerebral oedema in Sweden--a 2-year paediatric population study. Diabet Med 2007; 24: 1080-1085. https://doi.org/10.1111/j.14645491.2007.02200.x

26. Kong YH, Kim MS, Lee DY. Comparison of the prevalance of islet autoantibodies according to age disease duration in patients with type 1 diabetes mellitus. Ann Pediatr Endocrinol Metab 2013; 18: 6570. https://doi.org/10.6065/apem.2013.18.2.65

27. Acar S, Gören Y, Paketçi A, et al. Changes in the frequency of diabetic ketoacidosis in type i diabetes mellitus cases at diagnosis: a fifteen-year single center experience. J Pediatr Res 2017; 4: 143-148. https://doi.org/10.4274/jpr.16768

28. Larkin ME, Lorenzi GM, Bayless M, Cleary PA, Barnie A, Golden E. Evolution of the study coordinator role: the 28-year experience in diabetes control and complications trial/epidemiology of diabetes interventions and complications (DCCT/ EDIC). Clin Trials 2012; 9: 418-425. https://doi. org/10.1177/1740774512449532
29. Jackson W, Hofman PL, Robinson EM, Elliot RB Pilcher CC, Cutfield WS. The changing presentation of children with newly diagnosed type 1 diabetes mellitus. Pediatr Diabetes 2001; 2: 154-159. https:// doi.org/10.1034/j.1399-5448.2001.20403.x

30. Manuwald U, Schoffer O, Hegewald J, et al. Ketoacidosis at onset of type 1 diabetes in children up to 14 years of age and the changes over a period of 18 years in Saxony, Eastern-Germany: a population based register study. PloS One 2019; 14: e0218807. https://doi.org/10.1371/journal.pone.0218807 